\title{
Working Memory for Complex Scenes: Age Differences in Frontal and Hippocampal Activations
}

\author{
Denise C. Park ${ }^{1}$, Robert C. Welsh ${ }^{2}$, Christy Marshuetz ${ }^{3}$, \\ Angela H. Gutchess ${ }^{2}$, Joseph Mikels ${ }^{2}$, Thad A. Polk ${ }^{2}$, \\ Douglas C. Noll ${ }^{2}$, and Stephan F. Taylor ${ }^{2}$
}

\begin{abstract}
Age differences in frontal and hippocampal activations in working memory were investigated during a maintenance and subsequent probe interval in an event-related fMRI design. Younger and older adults either viewed or maintained photographs of real-world scenes (extended visual or maintenance conditions) over a 4-sec interval before responding to a probe fragment from the studied picture. Behavioral accuracy was largely equivalent across age and conditions on the probe task, but underlying neural activations differed. Younger but not older adults showed increased left anterior hippocampal activations in
\end{abstract}

\section{INTRODUCTION}

Performance on working memory tasks continuously declines with age (Park et al., 1996; Park \& Hedden, 2002; Park et al., 2002). Despite this simple linear decline in performance, the underlying neural activations associated with age-related changes in working memory function present a considerably more complex picture. There is evidence for both qualitative and quantitative differences between old and young in neural activation in the frontal cortex on working memory tasks (Reuter-Lorenz et al., 2000; Rypma \& D'Esposito, 2000, Rypma, Prabhakaran, Desmond, \& Gabrieli, 2001). One pattern of findings is that older adults are more likely to show bilateral patterns of neural recruitment in the prefrontal cortex, whereas young adults tend to show lateralized recruitment patterns. For example, Reuter-Lorenz et al. (2000), using positron emission tomography (PET), reported that older adults evidenced more bilateral activation of the dorsolateral prefrontal cortex for visuospatial and verbal working memory tasks, whereas younger adults showed hemisphere-specific activations. Cabeza (2001) notes similar patterns of finding for episodic retrieval, and suggests that greater neural activation in the frontal cortex is a compensatory response to decreased neural efficiency with age, although Logan, Sanders, Snyder, Morris, and Buckner

\footnotetext{
${ }^{1}$ University of Illinois at Urbana-Champaign, ${ }^{2}$ University of Michigan, ${ }^{3}$ Yale University
}

the extended visual compared with the maintenance condition. On the subsequent probe interval, however, older adults showed more left and right inferior frontal activations than younger adults. The increased frontal activations at probe in older adults may have been compensatory for the decreased hippocampal activations during maintenance, but alternatively could have reflected the increased difficulty of the probe task for the older subjects. Thus, we demonstrate qualitatively different engagement of both frontal and hippocampal structures in older adults in a working memory task, despite behavioral equivalence.

(2002) present evidence suggesting that the increased neural activation is dysfunctional.

Rypma and D'Esposito (2000) and Rypma et al. (2001), using sensitive event-related functional imaging designs, have reported somewhat different patterns of findings for frontal areas on working memory tasks. Rypma and D'Esposito (2000) observed increased bilateral dorsal lateral prefrontal recruitment in old and not young adults on a supra-span letter maintenance tasks, but equivalent activation with age of ventral lateral cortex. The increased activations occurred only at retrieval. In a related study, Rypma et al. (2001) again observed equivalent ventral lateral activations with age in the prefrontal cortex on a supra-span letter task, but reported as well, that young adults showed heightened dorsal lateral prefrontal activations, whereas old adults showed increased rostral lateral activations. Thus, in this study, the two age groups used different areas of the frontal cortex to perform the same task. Rypma and D'Esposito (2000) and Rypma et al. (2001) concluded that ventral lateral pathways used for storage and/or maintenance at encoding are intact with age, but higher-order processing that is required by retrieval from working memory is compromised with age.

Although the role of the frontal cortex is well documented in working memory function, it is less recognized that the hippocampus plays an important role in working memory. There are studies of young adults implicating the hippocampus in both picture maintenance (Ranganath \& D'Esposito, 2001), as well as in building strong 
memories during encoding of pictures (Brewer, Zhao, Desmond, Glover, \& Gabrieli, 1998) and words (Wagner et al., 1998). Cohen et al. (1999) have argued that the hippocampus plays a central role in relational processing of elements within a complex, meaningful scene as well as in binding of targets to context. Cohen et al. view the hippocampus as the machinery that allows one to make sense of the environment, and suggest that relational processing is automatic and obligatory, and that the hippocampus is always engaged for processing of complex pictures. They suggest that the hippocampus will not be engaged when encoding simple stimuli, noting that "when there are no objects or just very few objects as input to the system, as when the stimuli consist of just a fixation cross or noise patterns, there is little or no binding that can be done; the hippocampal system will be less active and will show little or no activation in functional imaging studies" (p. 95).

Little is known about how age affects hippocampal activity in working memory. We focus in the present study on the role of the hippocampus in the processing of scenes in working memory, as a method for elucidating the role of the hippocampus. On one hand, older adults would appear to be efficient at relational processing, given extensive evidence that immediate recognition of complex, meaningful scenes is age-invariant (Smith, Park, Cherry, \& Berkovsky, 1990; Park, Puglisi, \& Smith, 1986). These data would suggest equivalent activation of hippocampal structures in processing meaningful scenes between old and young. On the other hand, there are four lines of evidence that lead to the expectation that older adults will be less efficient in relational processing and hippocampal function. First, there is clear evidence that older adults are deficient in explicitly binding targets to context (Chalfonte \& Johnson, 1996; Park, Puglisi, \& Lutz, 1982; Park \& Puglisi, 1985). Second, there is evidence that there is less activation in the left anterior hippocampus in old compared with young adults on trials where binding of objects to location information was required (Mitchell, Johnson, Raye, \& D'Esposito, 2000), and third, hippocampal volume declines with age which likely causes diminished function (Raz, 2000). Finally, Park, Royal, Dudley, and Morrell (1988) observed equivalent picture recognition of meaningful scenes in old and young adults at immediate recognition, but reported much steeper declines in delayed memory for the scenes in older adults at intervals ranging from 2 days to 1 month. This finding of a more fragile scene memory over time with age is suggestive of decreased hippocampal involvement in older adults at encoding that is not apparent on easier immediate recognition tasks.

In the present study, we examine jointly the role of frontal and hippocampal structures in a working memory task as a function of age. We employ an event-related design that allows us to parse activations in these sites into encoding, maintenance, and retrieval phases (Rypma \& D'Esposito, 2000; Rypma et al., 2001). As noted earlier, Rypma and D'Esposito (2000) used an eventrelated fMRI design on a working memory task, focusing on frontal activations in young and old adults. They observed age differences in activations primarily in the retrieval phase of the task, with older adults evidencing more activation of the dorsolateral prefrontal cortex than younger adults when load was higher. They reported no age differences in activations of storage-based ventral lateral prefrontal cortex. In a related event-based design that focused on the hippocampus, Ranganath and D'Esposito (2001) observed right anterior hippocampal engagement in the maintenance of meaningful faces, although they did not explore age differences.

In the present study, we required subjects to maintain complex meaningful pictures across a retention interval or to engage in passive viewing of a picture and then immediately after each item was presented, respond to a probe. We chose to study complex pictures because there is a wealth of behavioral data suggesting that older adults show preserved function relative to younger adults in encoding and immediate memory for complex, meaningful pictures (Smith et al., 1990; Park et al., 1986; Park, Puglisi, Smith, \& Dudley, 1987). Equivalent, nonceiling behavioral performance on a task between age groups provides ideal conditions for the interpretation of age-differences in neural activations (Park, Polk, Mikels, Taylor, \& Marshuetz, 2001; Reuter-Lorenz, Marshuetz, Jonides, \& Smith, 2001; Grady, 1998; Cabeza et al., 1997), and we believed the use of meaningful pictures would provide this solid behavioral foundation for the imaging work. We used an event-related design because it permitted us to understand the joint operation of frontal and medial temporal structures in younger and older adults across encoding, maintenance, and probe as a function of age. Finally, we contrasted passive pictorial encoding with pictorial maintenance to determine whether the more effortful maintenance task would result in increased neural activation in older relative to younger adults.

We hypothesized that younger adults would show more hippocampal activation during a maintenance interval (as observed by Ranganath \& D'Esposito, 2001 for faces) than older adults, but that older adults would show greater frontal activations at probe (as observed by Rypma et al., 2001), possibly as a compensatory response to decreased hippocampal activation. We also hypothesized that we would see evidence for less lateralized responses in older compared with younger adults as observed by Reuter-Lorenz et al. (2000), Cabeza et al. (1997), and Grady et al. (1995).

The only data that we are aware of that examined maintenance of meaningful stimuli in young and old across a brief retention interval is the work of Grady and colleagues on memory for faces. Using a delayed matchto-sample task with a delay between 1 and $21 \mathrm{sec}$, younger adults evidenced more right prefrontal activations while the older adults showed more left prefrontal 
Figure 1. A schematic of the task for the extended visual and maintenance conditions.

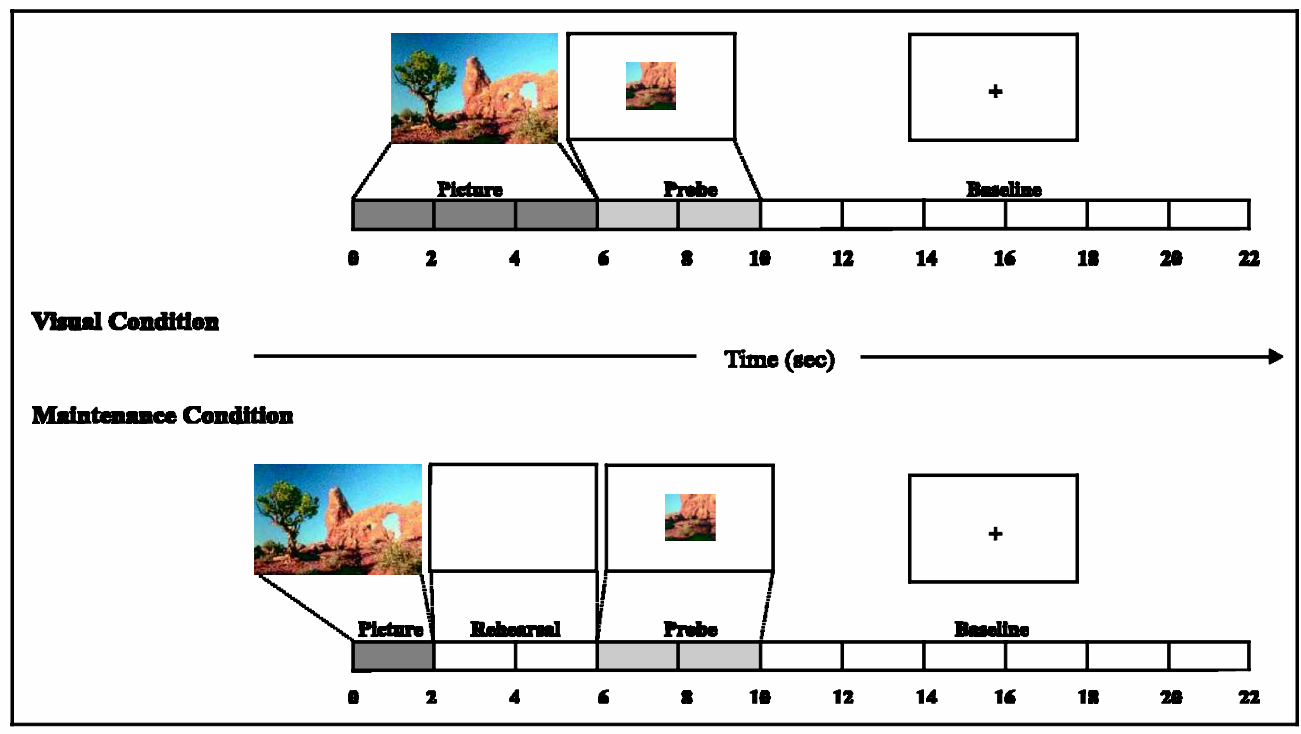

activations during the delay interval (Grady et al., 1998). McIntosh et al. (1999) focused on abstract pictures, and studied retention in working memory of abstract gratings that varied in spatial frequency for younger and older adults. Participants maintained the stimuli in memory for either 500 or $4000 \mathrm{msec}$ and then judged which pattern had a higher spatial frequency. Older adults recruited unique areas in the dorsolateral prefrontal cortex and medial temporal areas, compared

Table 1a. Behavioral Performance Data: Performance on Probe Task as a Function of Age and Condition

\begin{tabular}{lrrrrr}
\hline & \multicolumn{2}{c}{ Visual } & & \multicolumn{2}{c}{ Maintenance } \\
\cline { 2 - 3 } & Young & Old & & Young & Old \\
\hline Proportion correct & 83.71 & 82.67 & 81.63 & 80.43 \\
SD & 5.95 & 3.26 & 7.02 & 7.40 \\
Reaction Time & & & & \\
Hit & 1219 & 1716 & 1193 & 1646 \\
SD & 223 & 432 & 192 & 288 \\
Correct Rejection & 1544 & 1672 & & 1418 & 1471 \\
SD & 231 & 384 & & 171 & 330 \\
\hline
\end{tabular}

Table 1b. Behavioral Performance Data: Performance on Long-Term Memory Task as a Function of Age and Condition

\begin{tabular}{cccccc}
\hline & \multicolumn{2}{c}{ Visual } & & \multicolumn{2}{c}{ Maintenance } \\
\cline { 2 - 3 } \cline { 6 - 6 } & Young & Old & & Young & Old \\
\hline$A^{\prime}$ score $^{\mathrm{b}}$ & 0.80 & 0.73 & & 0.76 & 0.67 \\
\hline
\end{tabular}

${ }^{\mathrm{b}} A^{\prime}$ are calculated using high confidence responses only. with younger adults. All of these studies used PET, so it is difficult to know what phase of the task activated specific brain areas. Moreover, none of these studies examined the maintenance of meaningful scenes, as the present study does.

We presented 11 younger and 10 older adults with two different types of tasks in an event-related fMRI design, as shown in Figure 1. In the extended visual condition (top half of Figure 1), subjects viewed a complex scene for $6 \mathrm{sec}$, followed immediately by a picture fragment probe (4-sec interval). They were to press a key to indicate whether or not the probe fragment was part of the picture that they had just studied. Each trial was followed by a 12 -sec baseline period. In the maintenance condition (bottom half of Figure 1), subjects viewed a complex scene for $2 \mathrm{sec}$, followed by a 4 -sec rehearsal interval where they were instructed to maintain a mental image in their head of the picture they had just seen. After this 6-sec interval (2 sec of picture and $4 \mathrm{sec}$ of maintenance), they responded to a probe fragment (4-sec interval), followed by a 12 -sec baseline. Subjects judged whether the fragment was part of the picture they studied, as shown in Figure 1. Subjects received 48 trials of each type presented in pseudorandom order. Of particular interest in the present design was the 4-sec interval where, in the maintenance condition, subjects were maintaining the stimulus so they could make a perceptual judgment when the probe appeared, but in the extended visual condition, they passively viewed the picture until the probe appeared. Comparison of performance relative to baseline in these conditions permitted us to determine what neural structures were used to perform each task within each age group. Subjects also received an unexpected long-term memory test for the 96 pictures that was administered outside of the scanner, giving one of three button-press responses for targets (studied items) and lures (never-presented items): "yes, 
Table 2. Regions of Significant Activation during Task Periods

\begin{tabular}{|c|c|c|c|c|}
\hline \multirow[b]{2}{*}{ Lobe } & & \multicolumn{2}{|c|}{ MNI Coordinates } & \\
\hline & Activation Region & $x$ & $y$ & Voxel Number \\
\hline
\end{tabular}

\section{Maintenance Interval}

Extended Visual Condition > Visual Baseline

Occipital

Right, dorsal

12

5.06

502

36

$-78$

21

5.01

$27-57$

$-15$

3.85

Left, dorsal

$-33$

$-81$

4.45

417

$-27$

$-66$

3.74

$-42-81$

3.18

Cingulate

Anterior

15

3.98

130

$-6$

12

3.18

Temporal

Medial, posterior

$-27$

4.57

535

$-12$

$-21$

4.36

15

$-54$

$-12$

4.42

Maintenance > Maintenance Baseline

$\begin{array}{ll}\text { Cingulate } & \text { Anterior } \\ \text { Subcortex } & \text { Left hippocampus }\end{array}$

$-6$

$-12$

9

0

Maintenance > Visual

Parietal

Right

Left

Frontal

Left, orbital

Temporal

Right, lateral
63

66

$-60$

$-42$

$-27$

$-21$

$-27$

$-36$

66

60
$-54$

$-51$

12

$-15$

$-27$

$-39$

$-36$

$-48$

$-51$

$-54$

42

30

21

$-15$

$-36$
45

$-15$

$-12$

3

4.52

4.33

3.88

3.58
203

248

699

4.52

4.50

4.48

686

3.99

3.92

3.86

249

3.74

3.62

3.86

149

3.58

\section{Probe Interval $^{\mathrm{a}}$}

Visual Probe > Visual Baseline

$\begin{array}{lrrrrr}\text { Frontal } & \text { Left, motor } & -57 & -27 & 48 & 4.77 \\ & & -48 & -18 & 48 & 4.14 \\ & & -42 & -36 & 60 & 3.97 \\ \text { Cingulate } & \text { Anterior } & 0 & 12 & 48 & 3.96 \\ & & -3 & 12 & 33 & 3.71\end{array}$


Table 2. (continued)

\begin{tabular}{|c|c|c|c|c|c|c|}
\hline \multirow[b]{2}{*}{ Lobe } & \multirow[b]{2}{*}{ Activation Region } & \multicolumn{3}{|c|}{ MNI Coordinates } & \multirow[b]{2}{*}{$t$ score } & \multirow[b]{2}{*}{ Voxel Number } \\
\hline & & $x$ & $y$ & $z$ & & \\
\hline \multirow[t]{4}{*}{ Frontal } & \multirow[t]{2}{*}{ Left, inferior } & -42 & 18 & 15 & 4.35 & \multirow[t]{2}{*}{205} \\
\hline & & -57 & 18 & 21 & 3.73 & \\
\hline & \multirow[t]{2}{*}{ Right, inferior } & 60 & 15 & 27 & 3.98 & \multirow[t]{2}{*}{140} \\
\hline & & 66 & 18 & 21 & 3.79 & \\
\hline \multirow[t]{3}{*}{ Occipital } & \multirow[t]{3}{*}{ Right, dorsal } & 36 & -75 & 36 & 4.42 & \multirow[t]{3}{*}{227} \\
\hline & & 39 & -84 & 9 & 4.16 & \\
\hline & & 36 & -81 & 21 & 4.08 & \\
\hline \multirow[t]{3}{*}{ Subcortex } & \multirow[t]{3}{*}{ Left hippocampus } & -18 & -18 & -12 & 5.11 & \multirow[t]{3}{*}{871} \\
\hline & & -6 & -6 & -9 & 4.87 & \\
\hline & & -9 & -18 & -12 & 4.81 & \\
\hline
\end{tabular}

${ }^{\mathrm{a}} \mathrm{Visual}$ and maintenance subtractions yielded the same results, so we present the results only for the visual condition.

high confidence," “yes, low confidence," or "no, didn't study."

\section{RESULTS}

\section{Behavioral Data}

Behaviorally, both groups (younger and older) responded with equal accuracy (proportion correct for old and new items combined) to the probe in extended visual and maintenance trials (Table 1a). There were no significant main effects or interactions. With respect to reaction times, as depicted in Table 1 , young adults were faster than older adults, $F(1,19)=9.00, p<.01$ (marginal means are 1343.53 and 1626.26); and responses were quicker in the maintenance condition compared with the extended visual condition, $F(1,19)=7.27, p<.02$ (marginal means are 1431.82 and 1537.97). The faster responses in the maintenance condition may have occurred because subjects had to respond quickly before the maintained image degraded. In addition, there was a significant Age $\times$ Response type interaction that occurred, $F(1,19)=8.11, p<.02$, because young subjects were somewhat faster on hits than correct rejections (1206.13 vs. 1480.93), whereas older adults showed quicker responses to correct rejections (1571.29) compared to hits (1681.23).

With respect to performance on the long-term memory task that occurred outside of the scanner, an analysis of $A^{\prime}$ scores was conducted. A' is a measure of discriminability similar to $d$ ' that normalizes distributions of hits (saying "yes" to a presented item) and false alarms (saying "yes" to a never-seen item) such that it reflects actual memory better than hit rates alone. The A' scores appear in Table 1b. We excluded low confidence hits from this analysis and assessed accuracy only by examining responses to high confidence hits and high confidence false alarms to never-presented items, as in Brewer et al. (1998). An analysis of variance (ANOVA) with age (young/old) as a between-subjects variable and condition (visual/maintenance) as a withinsubject variable yielded a main effect of condition, $F(1,19)=7.76, p<.02$, such that pictures presented in the extended visual condition were better remembered than those presented in the maintenance condition (marginal means of 0.77 vs. 0.72 ). There was also a marginal effect of age, $F(1,19)=4.04, p<.06$, with the younger adults correctly remembering more than older adults (0.78 vs. 0.70$)$.

\section{Imaging Data}

\section{Group Analysis}

We first defined regions of interest by combining the data for the older and younger participants into a single random effects group analysis with condition (extended visual or maintenance) as a within-subject variable. This procedure provided us with an unbiased and conservative estimate of regions that were significantly activated when the subject groups were combined, and that we could then probe for age and condition differences. Table 2 presents results from three contrasts for the 4-sec maintenance interval: extended visual versus visual baseline, maintenance versus maintenance baseline, and maintenance versus extended visual. We initially examined our data for evidence of age differences in the magnitude of the hemodynamic response.

Figure 2 presents time-course data as a function of age and condition (maintenance and extended visual) for the left motor cortex resulting from the button press to each stimulus at probe. Visual inspection of the figure 


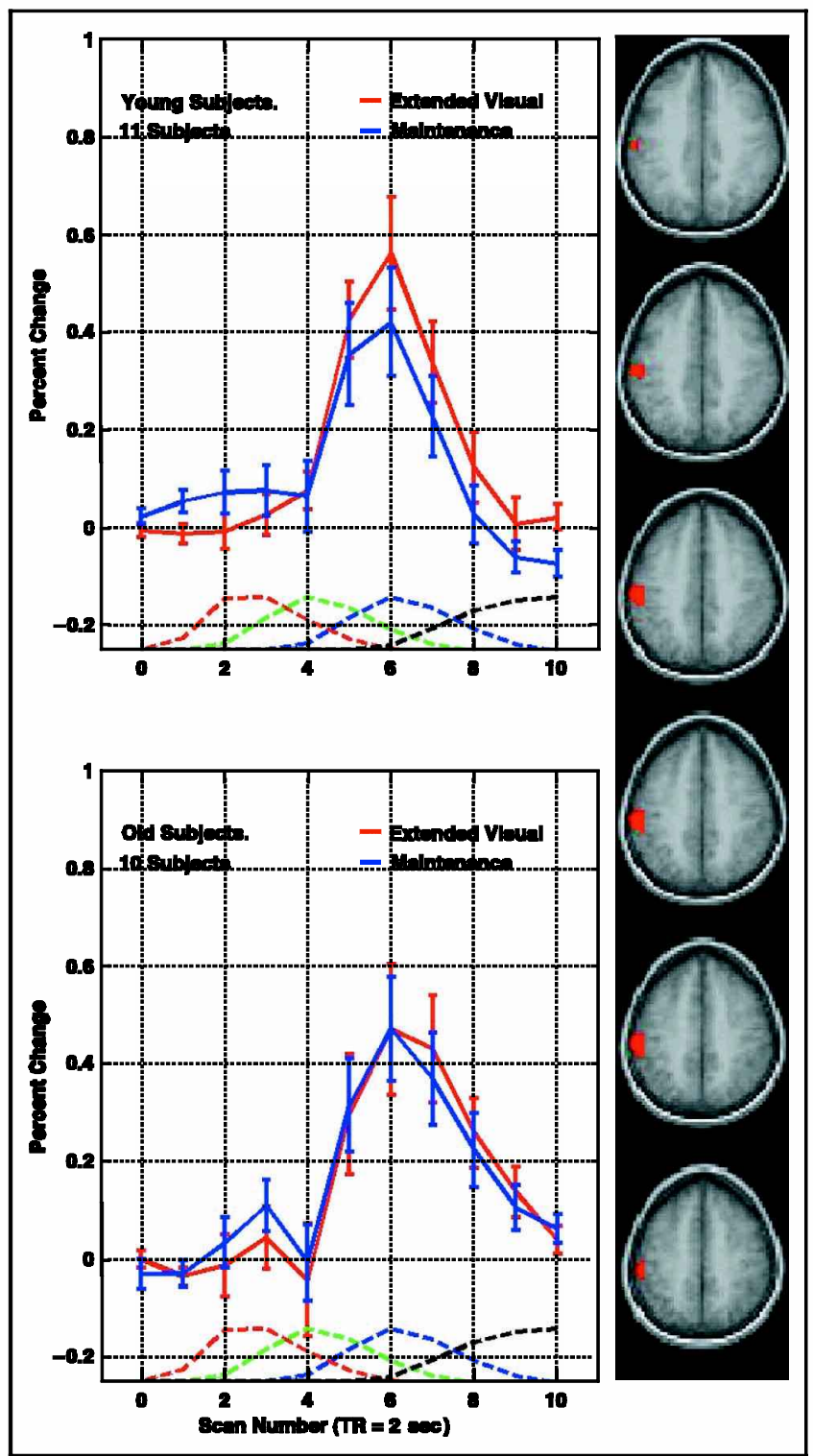

Figure 2. Group averaged time courses for the left motor cortex along with standard error. Young are shown on top and elderly on the bottom. The red indicates the extended visual condition and the blue the maintenance condition. The region of interest for the extracted data is shown in a group averaged normalized brain in red. Four model regressors are shown as dashed lines (initial 2-sec target as red; extended visual and maintenance as green; Probe as blue; and baseline as black), which were convolved with the standard HRF in SPM 99. Regions of interest were defined with the contrast of extended visual minus baseline. Individual responses were adjusted such that the mean of the first and the last two data points was zero.

indicates that there is little difference in the time courses as a function of age or condition. This impression was confirmed by a Huynh-Feldt corrected ANOVA that included age (young/old) and condition (extended visual/ maintenance), and time (Scans 3-7) as variables. The main effect of time was significant, $F(1.5,28.8)=26.17$, $p<.001$, but neither the age $(p>.65)$ nor condition $(p>.76)$ main effects were significant, nor was the interaction of age and condition $(p>.30)$. There was a weak trend towards a three-way interaction of age, time, and condition $(p>.10)$, caused largely by the slight difference in peak response between extended visual and maintenance conditions for young but not for old adults.

Because our hypotheses were focused on the medial temporal and frontal areas, we focused further analyses on these areas, using the MNI coordinates from the largest area of activation from the sites in Table 2 . Figure 3 presents time-course data for the left anterior hippocampal area. We analyzed the data by conducting an ANOVA on the points represented by Scans 3-7 (5 sec through $14 \mathrm{sec}$ of each trial) in each condition. A Huynh-Feldt corrected ANOVA that included age

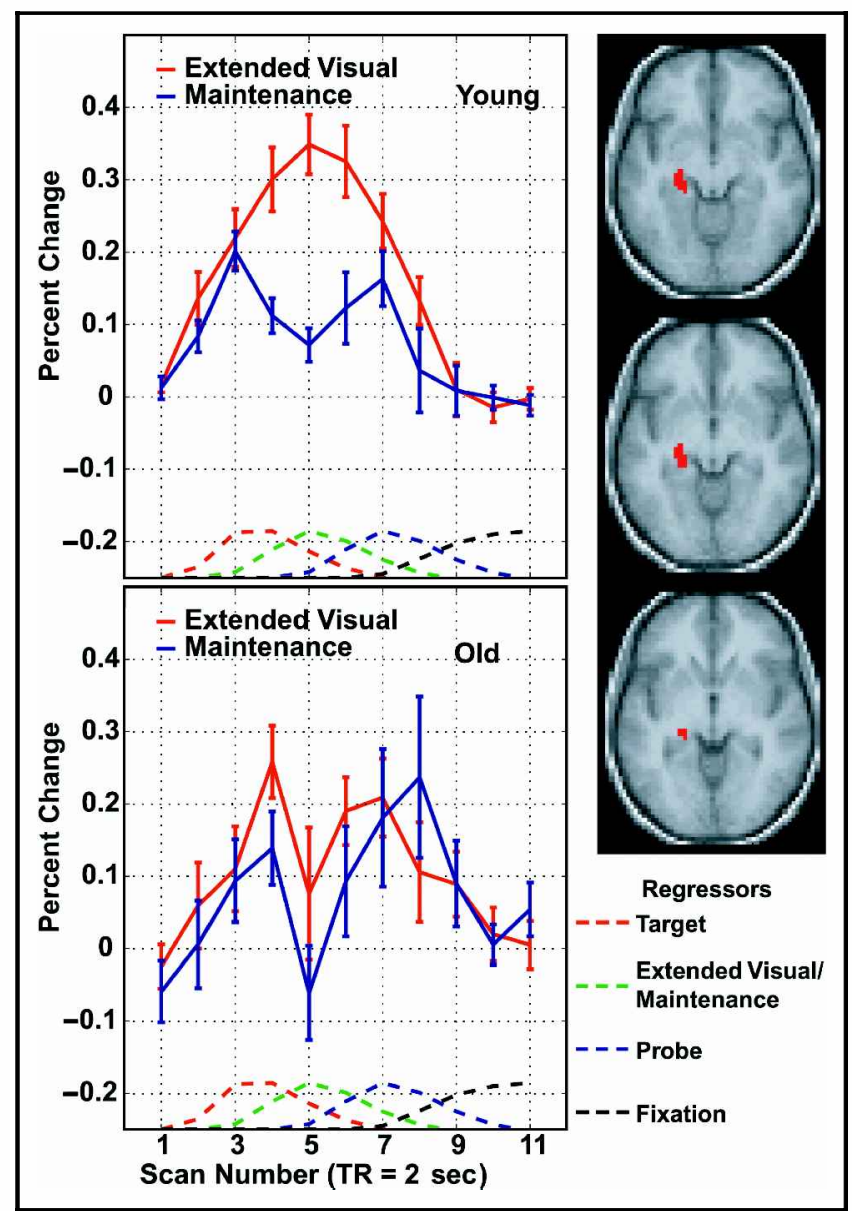

Figure 3. Group averaged time courses for the left hippocampus along with standard error. Young are shown on top and elderly on the bottom. The red indicates the extended visual condition and the blue the maintenance condition. The region of interest for the extracted data is shown in a group averaged normalized brain in red. Four model regressors are shown as dashed lines (initial 2-sec target as red; extended visual and maintenance as green; probe as blue; and baseline as black), which were convolved with the standard HRF in SPM 99. Regions of interest were defined with the contrast of extended visual minus baseline. Individual responses were adjusted such that the mean of the first and the last two data points was zero. 
(young/old), condition (extended visual and maintenance conditions), and time (Scans 3-7) yielded a main effect of condition, with more activation in the extended visual condition compared with the maintenance condition, $F(1,19)=48.67, p<.01$. However, this main effect was qualified by an Age $\times$ Condition interaction, $F(1,19)=4.83, p<.05$. The interaction occurred because hippocampal activation was more systematically differentiated in the young between the extended visual and maintenance conditions compared with the old. Finally, the Condition $\times$ Time interaction was significant, $F(3.3,62.8)=8.60, p<.001$, due to the U-shaped activation function in the maintenance condition for the young adults compared with the inverted U-shaped function in the extended visual condition.

Our results differ from Ranganath and D'Esposito (2001) in that they observed activation of the right anterior hippocampus during a maintenance interval after young subjects had encoded faces. We examined time courses for the other significant hippocampal areas presented in Table 2 and found no evidence for right hemisphere activation. However, we should note that even in the maintenance condition, paired $t$ tests comparing the average of Scans 3-7 against each subject's own baseline indicated that left hippocampal activation was above baseline for the young $[t(10)=5.80, p<.01]$. Although the average hippocampal activation for Scans 3-7 was not reliably different from baseline for the older adults $(p>.12)$, hippocampal activity was significantly greater than baseline at Scan $4[t(9)=2.73, p<.03]$, which suggests that older adults do recruit the hippocampus when the picture presentation ends and the maintenance task begins. Furthermore, the magnitude of the hippocampal activation in the maintenance condition did not differ between young and old $(p>.42)$ and there was no evidence for an Age $\times$ Time interaction $(p>$.19) based on a Huynh-Feldt corrected ANOVA that included age (young/old) and time (Scans $3-7)$ as variables. Note that this lack of age differences in the maintenance condition mirrors the motor cortex data in the similar activations exhibited by the young and old. Thus, our results are probably not dissimilar to those of Ranganath and D'Esposito (2001) in that the hippocampus is recruited in our maintenance task. We should also note that we conducted an anatomical analysis on a hippocampal ROI reported by Ranganath and D'Esposito (using an add-on SPM ROI toolbox developed by Poldrack and colleagues that can be found at http://spm-toolbox.sourceforge.net) and confirmed the findings reported from the group analysis.

We now focus on areas activated when the probe interval was analyzed (lower part of Table 2 ). The probe task was identical across conditions and the probe results did not differ for the extended visual and maintenance conditions. Of primary interest was the significant activation in the left and right inferior frontal cortex. Because we were particularly interested in bilateral activations in frontal areas, we examined average $t$ values bilaterally for the frontal cortex, based on regions noted in Table 2 that were significantly activated in either hemisphere during the probe interval. Mean $t$ values are presented in Figure 4 for activations in the inferior frontal gyrus at probe. An ANOVA of the $t$ values with age, condition, and hemisphere as variables yielded a main effect of age, $F(1,19)=5.05, p<.04$, with older adults showing more activation than younger adults. A comparison of the proportional increase in activation for older adults compared with young adults (using $t$ values displayed in Figure 4) yielded increased activations in older subjects, ranging from $43 \%$ in the maintenance condition for left hemisphere to a $63 \%$ increase in the extended visual condition in the right hemisphere. Although reaction times were somewhat longer in older adults at probe, the reaction time increases for old relative to young were $18.5 \%$ for the extended visual condition (29\% for hits and $8 \%$ for correct rejections) and $21 \%$ in the maintenance condition (38\% for hits and 4\% for correct rejections). Thus, increased time on task at probe cannot wholly explain the increased frontal activations in older adults. Additionally, there was a main effect of condition, $F(1,19)=16.23, p<.01$, with maintenance trials showing more frontal recruitment than extended visual trials ( $t$ values of 1.31 vs. 0.97), despite the finding that reaction times were longer in the extended visual condition. Thus, time on task cannot account for the greater activations in the maintenance condition. The expected Age $\times$ Laterality interaction was not significant $(p>.84)$. Although Figure 4 suggests that the older adults appeared to show more bilateral recruitment of the frontal regions than the younger adults, particularly in the extended visual condition, simple effects tests yielded no evidence for this. Correlational analyses between frontal and hippocampal activations were conducted

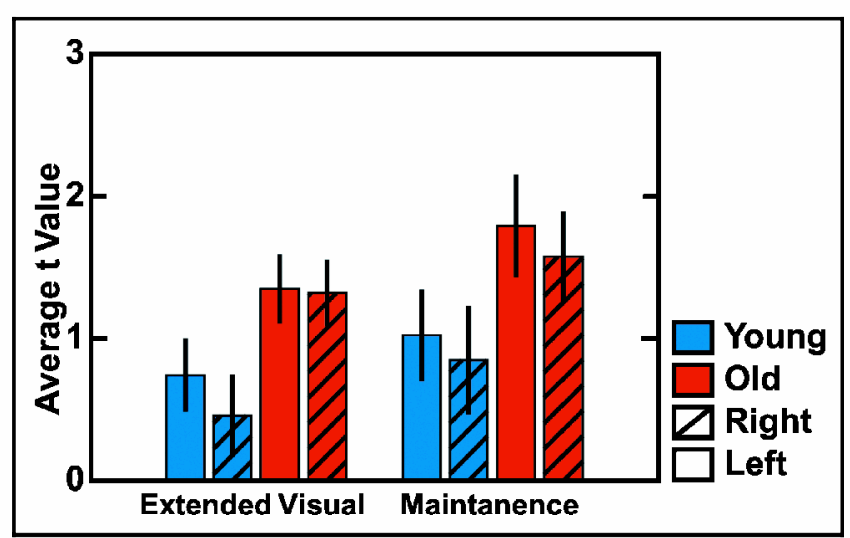

Figure 4. Group means and standard errors for average $t$-scores in the regions of interest defined by the 4-sec extended visual and maintenance conditions relative to the baseline condition. Young are shown in blue and elderly in red. Right and left visual cortices are identified with cross-hatched and solid bars. 
for young and old adults. The highest correlation was for old adults between the right frontal and left hippocampal region $(-.44)$, but this effect was far from significant $(p=.20)$.

\section{DISCUSSION}

We return to our original hypotheses regarding age and condition effects in the medial temporal and frontal cortex.

We focus first on the hippocampal areas. We observed the greatest activation in the left anterior hippocampus in young adults in the extended visual condition compared to the maintenance condition. Cohen et al. (1999) presented good evidence that the left anterior hippocampus is important for pictorial memory and that enhanced relational processing activates this area. Congruent with this finding, Brewer et al. (1998) reported activation in this area for remembered compared with forgotten pictures, whereas Wagner et al. (1998) found activation in this same area for remembered compared with forgotten words. Thus, it appears that the sustained presentation of the picture in the extended visual condition enhanced relational processing resulting in increased left anterior hippocampal activation for young adults. In support of this interpretation, the extended visual condition in the young adults showed the most left anterior hippocampal activation as well as the best out-of-scanner memory, as indicated in Table 1. It has been well-known for decades that meaningful scenes are extraordinarily wellremembered by young adults (Shepard, 1967). It may be that the basis for this memory is the enhanced relational processing (relative to the maintenance condition) that occurs spontaneously with complex meaningful scenes and the resulting increased hippocampal activations in young adults.

The finding of less left hippocampal activation in the maintenance condition suggests that a 2 -sec visual presentation of a picture may not be long enough for extended relational processing to occur. The fact that the hippocampal activation is above baseline in this condition for both old and young suggests some engagement of the hippocampus, which follows from Cohen et al.'s (1999) argument that relational processing via the hippocampus is automatic and obligatory. It also confirms the findings of Ranganath and D'Esposito who observed activation in the hippocampus in young adults for the maintenance of faces in working memory. We recognize that it would be interesting to contrast activations associated with remembered versus forgotten items as Brewer et al. (1998) and Wagner et al. (1998) did. We would expect to see stronger hippocampal activations for remembered compared to forgotten items. Due to the strong memory performance of participants, however, there were insufficient "forgotten" items to conduct such an analysis.
We now turn to the older adults. There are two interesting findings here. First, older adults did not show the magnitude of differentiation between extended visual and maintenance conditions in the left anterior hippocampus that young adults did, but did show above-baseline activation of the left anterior hippocampus in both conditions. The above-baseline hippocampal activation in the two conditions suggests that older adults show automatic, relational processing of pictorial stimuli, and that this automatic aspect of relational processing does not deteriorate. However, older adults did not show increased hippocampal function or better long-term memory for pictures presented for an extended period, whereas young adults did. This finding suggests that even normal elderly show some decline in hippocampal function compared to young adults, a finding congruent with Mitchell et al. (2000).

The older adult sample in this study was highly selected-subjects were on minimal medications, had nearly perfect Mini-Mental scores, and had higher verbal ability than the college students. Nevertheless, they showed a pattern of qualitatively different left anterior hippocampal activations in the present study. It is important to note when interpreting the results depicted in Figure 3 that long-term memory was not a requirement of the task-rather the task required maintenance of the image (most likely in ventral lateral streams) to prepare for an immediate response to the probe. Thus, one might argue that the decreased activations in the hippocampus do not represent a deficit in the elderly, particularly if the activations were important for long-term memory. It would be informative to compare incidental and intentional encoding conditions with respect to long-term memory, to determine if under intentional conditions, older adults engaged hippocampus and showed activations more similar to younger adults. It would be our expectation that relational processing is automatic and that intentionality would not alter hippocampal function. In fact, Logan et al. (2002) demonstrated that older adults showed large differences in frontal activations from young under intentional encoding conditions in a long-term memory paradigm, but equivalent activations when encoding was guided. A guided encoding manipulation might be more fruitful in understanding the control of hippocampal activations in older adults.

The present pattern of findings is also suggestive of a "dedifferentiated" hippocampal response in older adults. Although dedifferentiation of neural response with age has typically been discussed in the context of decreased laterality with age (Cabeza, 2001; Park et al., 2001; Reuter-Lorenz et al., 2001), dedifferentiation may be manifested in other ways. We suggest that dedifferentiation may also be evidenced by a less distinct neural signature with age in response to different experimental conditions. In this case, hippocampal response to the maintenance versus extended visual conditions was 
similar for old adults, but highly differentiated for young adults. Decreased differentiation of neural function with age could be a result of less reactivity to stimuli due to experience, but more likely represents declining neural efficiency.

The second hypothesis we tested was that older adults would show less lateralization and greater compensatory activations in frontal areas. Like Rypma and D'Esposito (2000) and Rypma et al. (2001), we did observe significantly greater activation in the left and right prefrontal cortex in older adults compared with younger adults at the time of the probe response. However, the increased activations we report occurred in the ventral prefrontal areas, rather than in the rostral or dorsal prefrontal cortex. Rypma and colleagues have suggested that the ventral prefrontal regions are ageinvariant storage-based structures, where differences in activation are not typically found, although their conclusions were drawn based on studies involving maintenance of simple letter strings. It may be that the amount of information contained in complex visual stimuli is sufficiently greater than that of letter strings so that increased age-related activations in ventral structures become apparent.

Like Rypma and D'Esposito (2000) and Rypma et al. (2001), neither younger nor older adults showed evidence of a significantly lateralized pattern of activation in a single hemisphere. Hence, our results are neutral with respect to the hemisphere asymmetry model of aging (Cabeza, 2001, 2002). Nevertheless, the increased frontal activation in older adults compared with younger adults is similar to findings reported by Reuter-Lorenz et al. (2000), Buckner, Snyder, Sanders, Raichle, and Morris (2000), and Cabeza et al. (1997). What is not clear is whether the increased frontal activations are compensatory for the decreased hippocampal activation observed during the extended visual condition, or whether the increased frontal activation is independent of the hippocampal activity. Correlational analyses did not yield significant relationships for either young or old between hippocampal activity at encoding and frontal at retrieval, but the small number of subjects makes the conclusion of independence far from certain. The fact that there was more recruitment of frontal areas in the maintenance compared with the extended visual condition suggests that the increased frontal activations reflected more retrieval effort. Only further research can resolve whether there is a hippocampal-frontal circuit associated with working memory for young adults whose neural signature is strong hippocampal activation during maintenance combined with weak frontal activation at retrieval (in contrast to older adults who show the opposite pattern). We should also note that median splits of high and low performers did not yield differences in patterns as in Rypma and D'Esposito (2000). However, the older adults were sufficiently selected that different patterns might be seen with a wider range of performance in a different sample.

In closing, the present study suggests diminished left hippocampal activations in older compared with younger subjects, with the pattern of findings tentatively pointing toward a failure to activate the hippocampus to engage in relational processing. These results suggest that medial temporal activations in extraordinarily healthy and cognitively intact older adults are less differentiated than those of young adults. Increased frontal activations at probe likely reflected the increased difficulty of the task for older adults, and may also reflect compensatory activations for decreased hippocampal activation.

\section{METHODS}

\section{Participant Demographics}

The participants were 11 ( 7 men, 4 women) younger adults with a mean age of 20.55 years and 10 ( 4 men, 6 women) older adults with an average age of 67.00 years, all right-handed, high-functioning, healthy, and neurologically normal, with no history of psychiatric

Table 3. Participant Characteristics, Demographics, and Neuropsychological Assessment

\begin{tabular}{lccc}
\hline & Young & Old & Significance $($ p) \\
\hline Age & $20.55(0.93)$ & $67.00(5.03)$ & .00 \\
Years of education & $14.73(0.65)$ & $15.30(1.77)$ & .33 \\
Mini-Mental State Exam & $29.70(0.67)$ & $29.10(0.99)$ & .13 \\
Shipley Vocabulary Task & $32.82(2.68)$ & $35.40(1.65)$ & .02 \\
Digit Span-Forward & $13.27(3.00)$ & $7.70(2.54)$ & .21 \\
Digit Span-Backward & $10.27(3.10)$ & $8.20(1.69)$ & .01 \\
Corsi Blocks-Forward & $9.55(1.75)$ & $7.10(1.45)$ & .09 \\
Corsi Blocks-Backward & $9.64(1.12)$ & .00 & .07 \\
\hline
\end{tabular}


disorder. Because most of the young volunteers were college students, the young adults had, on average, slightly fewer years of education (14.73 vs. 15.30), although this difference was not significant $(p=.09)$. The older adults' mean score on the Mini-Mental Status exam (Folstein, Folstein, \& McHugh, 1975) was a 29.10 and the young adults scored 29.70 . This difference was not significant and a perfect score on the Mini-Mental is 30 , so the older adults had exceptionally high mental functioning. A summary of demographics appears in Table 3 .

\section{Procedure}

Subjects participated in two sessions: one practice session to gain familiarity with the task and to complete neuropsychological testing, and one scanning session on a separate day. Subjects were paid between US $\$ 75$ and US $\$ 125$ for their participation depending on the length of the session. The study was approved by the University of Michigan Medical School Institutional Review Board.

\section{Practice Session}

In the practice session, subjects performed two blocks of practice on the experimental cognitive task. They received written and oral instructions that placed equal emphasis on speed and accuracy. In addition to practice on the experimental task, they were screened using a neuropsychological battery described below. The practice session lasted approximately $1.5 \mathrm{hr}$. Subjects gave full written consent.

\section{Neuropsychological Assessment}

We used a number of tasks to characterize our older and younger adults' baseline cognitive abilities: Forward and Backward Digit Span (from the Wechsler Adult Intelligence Scale-III; Wechsler, 1997a), Forward and Backward Corsi Blocks (from the Wechsler Memory Scale-III; Wechsler, 1997b), Shipley Vocabulary Test (from the Shipley Institute of Living Scale; Shipley, 1986). With the exception of the Shipley vocabulary test, the younger adults performed better on all of these measures, although not all these differences were significant (see Table 3).

\section{fMRI Session}

Subjects participated in six runs. Each run contained eight trials for each condition (visual, maintenance), in random order, for a total number of 48 trials in each condition. Runs lasted $6 \mathrm{~min}$ and $44 \mathrm{sec}$ each. Prior to the functional runs, both T1- and T2-weighted structural images were acquired. In total, the fMRI session lasted approximately $2 \mathrm{hr}$. The task was presented with a
Macintosh G3 using PsyScope (Cohen, MacWhinney, Flatt, \& Provost, 1993). Stimuli were back-projected onto a screen outside of the scanner, which subjects viewed through custom-made goggles that corrected each subject's visual acuity to 20/20.

\section{Experimental Cognitive Task}

Stimuli were digitized photographs chosen from several commercial (Corel) databases of color images. All images used in scanning were outdoor, natural landscapes and all practice images were indoor scenes. None contained humans or animals.

In both conditions, subjects saw a target image followed by a probe image, to which they had to respond negatively or positively by key-press with either the index finger or the middle finger, respectively, of the right hand. For both the maintenance and extended visual conditions, the proportion of positive and negative probes was 50\%. Positive probes were fragments of the original pictures containing one-sixth of the image (to scale) presented in the center of the screen. Negative probes consisted of picture fragments, also onesixth of the size of the target image, from other pictures not used elsewhere in the experiment. These negative probes were matched to the targets for color, content, texture, and brightness.

Trials in the visual condition consisted of the presentation of a target image for $6 \mathrm{sec}$, during which time subjects were instructed to study and form a mental representation of the stimulus. Immediately following presentation of the target image, subjects were presented with a probe image for $4 \mathrm{sec}$ and had to respond to the question, "Was this probe part of the image you just saw?" Following presentation of the probe, a 12 -sec baseline period occurred, during which subjects were instructed to rest. The baseline period served as a control condition and gave ample time for the hemodynamic response to return to baseline (Aguirre \& D'Esposito, 1999).

The maintenance condition contained the same events as the visual condition, with one exception. Recall that in the extended visual condition, the target appeared for $6 \mathrm{sec}$. In the maintenance condition, the target appeared for $2 \mathrm{sec}$, followed by a 4 -sec period during which subjects maintained a mental image until the probe appeared. Since trials occurred randomly, subjects did not know which type of trial would occur and presumably engaged in the same processes up until the point at which the stimulus disappeared.

Once subjects completed the probe judgment task for all 96 pictures, subjects received a surprise long-term memory test outside of the scanner after a 20-min delay. Subjects indicated by a key-press whether or not they had studied the item previously, responding "yes—high confidence," "yes—low confidence," or "no—not studied." Their memory was tested on all 96 studied pictures 
(targets) in addition to 72 similar, unstudied pictures (lures). A' scores are shown in Table $1 \mathrm{~b}$.

\section{Image Acquisition and Analysis}

\section{Acquisition}

Subjects participated in six fMRI runs (1.5 Tesla GE LX, BOLD sensitive gradient-echo EPI, TR $=2000 \mathrm{msec}$, TE $=$ $40 \mathrm{msec}, \mathrm{FOV}=240$ by $150 \mathrm{~mm}$ ) while performing the cognitive tasks, for a total of 528 scans per condition. Slices were $6 \mathrm{~mm}$ thick, 128 by 80 matrix (in-plane resolution 1.875 by $1.875 \mathrm{~mm}$ ), acquired at 14 interleaved contiguous locations parallel to the anterior commissure-posterior commissure line. Slices covered most of the cortex, but did not cover the cerebellum for a majority of the subjects, and the inferior occipital cortex in some subjects. After completion of the cognitive portion of the study, a high-resolution 3-D-SPGR $(0.9375 \mathrm{~mm}$ in-plane resolution, $1.5-\mathrm{mm}$-thick slice) volume was acquired. This scan served as the basis for normalization to a standard stereotactic system.

\section{Random-Effects Analysis}

All fMRI data were analyzed using SPM99 (Wellcome Department of Cognitive Neurology, London) implemented in MATLAB (Mathworks, Sherborn, MA). First, we employed intrasubject registration to correct motion between scans. Second, we transformed each subject's data to a stereotaxic system (MNI Brain) by taking the following steps: (1) we registered each subjects' mean realigned EPI-bold data to his or her volumetric 3-D structural scan; (2) the registered volumetric scan was then normalized to the standard MNI space; (3) the transformation parameters obtained during normalization of the volumetric scan were then applied to the registered realigned functional images, which were resampled to 3-mm isotropic voxels.

Following normalization, we applied intrasubject spatial smoothing to the data using a 5-mm FWHM gaussian filter. For each subject, we generated $t$-statistic maps, resulting in contrast images for each of our contrasts-ofinterest. A model of two main trial types (visual and maintenance) was implemented, with each trial being modeled with four regressors of interest. Fixed-effect analysis was done using a 120-sec high-pass filter. Finally, we applied intersubject smoothing, using an 8-mm FWHM gaussian filter and tested for group activation utilizing the random-effects method (Friston, Holmes, Price, Büchel, \& Worsley, 1999; Holmes \& Friston, 1998).

The random effects analysis employs a single sample $t$ test for a contrast of interest and produces estimates of the size and intensity of activations using gaussian random field theory (Worsley et al., 1996). The threshold for voxels to enter the analysis was set to an uncorrected $p$ value $<.001$ for all contrasts. In all cases, cluster-level $p$ values reported here reflect a correction for multiple comparisons.

\section{Event-Related Analysis}

Our event-related analysis was based on a number of regions-of-interest defined using the following method: First, we performed a random-effects analysis that included both the younger and the older subjects in a onesample $t$ test. These results were thresholded at $p=0.001$ (uncorrected) for the purposes of data reduction. Second, the results were visually superimposed on an anatomical template image that was created using the average of a random sample of 12 volumetric scans from our population of younger and older adults. Third, regions of interest were chosen by defining the most highly activated areas in anatomically constrained regions.

After defining regions of interest, we extracted each individual subject's time-course data from those regions using the co-registered and normalized fMRI images (but not smoothed). Time-course data were then high-pass filtered with a 110-sec cutoff. We then selectively averaged each subject's time-course data together based on the trial type (extended visual or maintenance), to produce a mean time course for each contrast of interest for each subject. Finally, we averaged each subject's time course with the others from that group, to produce a mean time course for each region for each group (younger, older). Plots depict the average time course (and standard error) for each group.

\section{Laterality Analysis}

The regions of interest defined in the extraction of the time course served as the basis for the laterality analysis of the inferior frontal cortex. For a given contrast of interest, the regions of interest defined on the left and right sides of the brain were mirror reflected about the brain midline. These were then formed in a union and produced a laterality mask. The mask was explicitly zeroed about the midline of the brain to form a left and right brain mask. These left and right brain masks were then applied to the individual subject statistical maps ( $t$ maps) for each contrast of interest and the $t$ score was averaged over the mask. The resulting average $t$ statistic and standard error in the group $t$ statistic are shown in Figure 4.

\section{Acknowledgments}

This research was supported by National Institute on Aging Neuroscience and Neuropsychology Program Grant R01AGO6265-15.

Reprint requests should be sent to Denise C. Park, The Beckman Institute, University of Illinois, $405 \mathrm{~N}$. Mathews, Urbana, IL 61801 USA, or via e-mail: denisep@uiuc.edu. 
The data reported in this experiment have been deposited in the fMRI Data Center (http://www.fmridc.org). The accession number is $2-2003-113 Q X$.

\section{REFERENCES}

Aguirre, G. K., \& D'Esposito, M. (1999). Experimental design for brain $f M R I$. In C. T. W. Moonsen \& P. A. Bandettini (Eds.), Functional fMRI. Berlin: Springer-Verlag.

Brewer, J. B., Zhao, Z., Desmond, J. E., Glover, G. H., \& Gabrieli, J. D. E. (1998). Making memories: Brain activity that predicts how well visual experience will be remembered. Science, 281, 1185-1187.

Buckner, R. L., Snyder, A. Z., Sanders, A. L., Raichle, M. E., \& Morris, J. C. (2000). Functional brain imaging of young, nondemented and demented older adults. Journal of Cognitive Neuroscience, 12, 24-34.

Cabeza, R. (2001). Functional neuroimaging of cognitive aging. In R. Cabeza \& A. Kingstone (Eds.), Handbook of functional neuroimaging of cognition. Cambridge: MIT Press.

Cabeza, R. (2002). Hemispheric asymmetry reduction in older adults: The HAROLD model. Psychology and Aging, 17, 85-100.

Cabeza, R., Grady, C. L., Nyberg, L., McIntosh, A. R., Tulving, E., Kapur, S., Jennings, J. M., Houle, S., \& Craik, F. I. M. (1997). Age-related differences in neural activity during memory encoding and retrieval: A positron emission tomography study. Journal of Neuroscience, 17, 391-400.

Chalfonte, B. L., \& Johnson, M. K. (1996). Feature memory and binding in young and older adults, Memory and Cognition, 24, 403-416.

Cohen, J. D., MacWhinney, B., Flatt, M., \& Provost, J. (1993). PsyScope: A new graphic interactive environment for designing psychology experiments. Behavioral Research Methods, Instruments, and Computers, 25, 257-271.

Cohen, N. J., Ryan, J., Hunt, C., Romine, L., Wszalek, T., \& Nash, C. (1999). Hippocampal system and declarative (relational) memory: Summarizing the data from functional neuroimaging studies. Hippocampus, 9, 83-98.

Folstein, M. F., Folstein, S. E., \& McHugh, P. R. (1975). Mini-Mental state: A practical method for grading the cognitive state of patients for the clinician. Journal of Psychiatry Research, 12, 189-198.

Friston, K. J., Holmes, A. P., Price, C. J., Büchel, C., \& Worsley, K. J. (1999). Multisubject fMRI studies and conjunction analyses. Neuroimage, 10, 385-396.

Grady, C. L. (1998). Functional brain imaging and age-related changes in cognition. Biological Psychology, 54, 259-281.

Grady, C. L., McIntosh, A. R., Bookstein, F., Horwitz, B., Rapoport, S. I., \& Haxby, J. V. (1998). Age-related changes in regional cerebral blood flow during working memory for faces. Neuroimage, 8, 409-425.

Grady, C. L., McIntosh, A. R., Horwitz, B., Maisog, J. M., Ungerleider, L. G., Mentis, M. J., Pietrini, P., Schapito, M. B., \& Haxby, J. V. (1995). Age-related reductions in human recognition memory due to impaired encoding, Science, 269, 218-221.

Holmes, A. P., \& Friston, K. J. (1998). Generalisability, random effects and population inference. Neuroimage, 7 , S754.

Logan, J. M., Sanders, A. L., Snyder, A., Morris, J. C., \& Buckner, R. L. (2002). Under-recruitment and non-selective recruitment: Dissociable neural mechanisms associated with aging. Neuron, 33, 827-840.

McIntosh, A. R., Sekuler, A. B., Penpeci, C., Rajah, M. N., Grady, C. L., Sekuler, R., \& Bennett, P. J. (1999). Recruitment of unique neural systems to support visual memory in normal aging. Current Biology, 9, 1275-1278.

Mitchell, K. J., Johnson, M. K., Raye, C. L., \& D'Esposito, M. (2000). fMRI evidence of age-related hippocampal dysfunction in feature binding in working memory. Cognitive Brain Research, 10, 197-206.

Park, D. C., \& Hedden, T. (2002). Working memory and aging. In M. Naveh-Benjamin, M. Moscovitch, \& H. L. Roediger III (Eds.), Perspectives on human memory and cognitive aging: Essays in honour of Fergus Craik. East Sussex, UK: Psychology Press.

Park, D. C., Lautenschlager, G., Hedden, T., Davidson, N. S., Smith, A. D., \& Smith, P. K. (2002). Models of visuospatial and verbal memory across the adult life span. Psychology and Aging, 17, 299-320.

Park, D. C., Polk, T. A., Mikels, J. A., Taylor, S. F., \& Marshuetz, C. (2001). Cerebral aging: Integration of brain and behavioral models of cognitive function. Dialogues in Clinical Neuroscience: Cerebral Aging, 3, 151-165.

Park, D. C., \& Puglisi, J. T. (1985). Older adults' memory for the color of matched pictures and words. Journal of Gerontology, 40, 198-204.

Park, D. C., Puglisi, J. T., \& Lutz, R. (1982). A spatial memory in older adults: Effects of intentionality. Journal of Gerontology, 37, 330-335.

Park, D. C., Puglisi, J. T., \& Smith, A. D. (1986). Memory for pictures: Does an age-related decline exist? Psychology and Aging, 1, 11-17.

Park, D. C., Puglisi, J. T., Smith, A. D, \& Dudley, W. N. (1987). Cue utilization and encoding specificity in picture recognition by older adults. Journal of Gerontology, 42, 423-425.

Park, D. C., Royall, D., Dudley, W., \& Morrell, R. (1988). Forgetting of pictures over a long retention interval in old and young adults. Psychology and Aging, 3, 94-95.

Park, D. C., Smith, A. D., Lautenschlager, G., Earles, J., Frieske, D., Zwahr, M., \& Gaines, C. (1996). Mediators of long-term memory performance across the life-span. Psychology and Aging, 11, 621-637.

Ranganath, C., \& D'Esposito, M. (2001). Medial temporal lobe activity associated with active maintenance of novel information. Neuron, 31, 865-873.

Raz, N. (2000). Aging of the brain and its impact on cognitive performance: Integration of structural and functional findings. In F. I. M. Craik \& T. A. Salthouse (Eds.), Handbook of aging and cognition (pp. 1-90). Mahwah, NJ: Erlbaum.

Reuter-Lorenz, P. A., Jonides, J., Smith, E., Hartley, A., Miller, A., Marshuetz, C., \& Koeppe, R. (2000). Age differences in the frontal lateralization of verbal and spatial working memory revealed by PET. Journal of Cognitive Neuroscience, 12, 174-187.

Reuter-Lorenz, P. A., Marshuetz, C., Jonides, J., \& Smith, E. E. (2001). Neurocognitive ageing of storage and executive processes. European Journal of Cognitive Psychology, 13, 257-278.

Rypma, B., \& D'Esposito, M. (2000). Isolating the neural mechanisms of age-related changes in human working memory. Nature Neuroscience, 3, 509-515

Rypma, B., Prabhakaran, V., Desmond, J. E., \& Gabrieli, J. D. (2001). Age differences in prefrontal cortical activity in working memory. Psychology and Aging, 16, 371-384.

Shepard, R. N. (1967). Recognition memory for words, sentences, and pictures. Journal of Verbal Learning and Verbal Behavior, 6, 156-163.

Shipley, W. C. (1986). Shipley Institute of Living Scale. Los Angeles: Western Psychological Services.

Smith, A. D., Park, D. C., Cherry, K., \& Berkovsky, K. (1990). 
Age differences in memory for concrete and abstract pictures. Journal of Gerontology, 35, 205-209.

Wagner, A. D., Schacter, D. L., Rotte, M., Koutstaal, W., Maril, A., Dale, A. M., Rosen, B. R., \& Buckner, R. L. (1998). Building memories: Remembering and forgetting of verbal experiences as predicted by brain activity. Science, 281, 1188-1191.

Wechsler, D. (1997). Wechsler Adult Intelligence Scale-
Third Edition. San Antonio, TX: The Psychological Corporation.

Wechsler, D. (1997). Wechsler Memory Scale-Third Edition. San Antonio, TX: The Psychological Corporation.

Worsley, K. J., Marrett, S., Neelin, P., Vandal, A. C., Friston, K. J., \& Evans, A. C. (1996). A unified statistical approach for determining significant voxels in images of cerebral activation. Human Brain Mapping, 4, 58-73. 
Copyright of Journal of Cognitive Neuroscience is the property of MIT Press and its content may not be copied or emailed to multiple sites or posted to a listserv without the copyright holder's express written permission. However, users may print, download, or email articles for individual use. 